\title{
Terahertz dielectric response of photoexcited carriers in Si revealed via single-shot optical-pump and terahertz-probe spectroscopy
}

\author{
Yasuo Minami, Kohei Horiuchi, Kaisei Masuda, Jun Takeda, and Ikufumi Katayama ${ }^{a)}$ \\ Department of Physics, Graduate School of Engineering, Yokohama National University, Yokohama 240-8501, \\ Japan
}

(Received 3 September 2015; accepted 15 October 2015; published online 27 October 2015)

\begin{abstract}
We have demonstrated accurate observations of terahertz ( $\mathrm{THz}$ ) dielectric response due to photoexcited carriers in a Si plate via single-shot optical-pump and THz-probe spectroscopy. In contrast to conventional $\mathrm{THz}$ time-domain spectroscopy, this spectroscopic technique allows single-shot detection of the $\mathrm{THz}$ response of materials at a given delay time between the pump and $\mathrm{THz}$ pulses, thereby sufficiently extending the time interval between the pump pulses. As a result, we can accurately measure the dielectric properties of materials, while avoiding artifacts in the response caused by the accumulation of long-lived photoexcited carriers. Using our single-shot scheme, the transmittance of a Si plate was measured in the range of $0.5-2.5 \mathrm{THz}$ with different pump fluences. Based on a Drude model analysis, the optically induced complex dielectric constant, plasma frequency, and damping rate in the THz region were quantitatively evaluated. (C) 2015 AIP Publishing LLC. [http://dx.doi.org/10.1063/1.4934697]
\end{abstract}

Silicon $(\mathrm{Si})$ has attracted much attention for use in various kinds of semiconductor devices, such as storage devices, optical sensors, and thermal sensors. ${ }^{1-3}$ For each of these applications, a fundamental understanding of carrier dynamics is extremely important for improving the performance of the devices; for this reason, optical-pump and optical-probe experiments have been performed to reveal the carrier dynamics in $\mathrm{Si}^{4-7}$ These prior experimental results have disclosed the picosecond to nanosecond decay properties of photoexcited carriers in the infrared to visible light region. The existence of long-lived carriers, which might be in deep energy levels, was also suggested in previous studies. ${ }^{8,9}$ Recent progress in terahertz time-domain spectroscopy (THz-TDS) allows us to access the dielectric response due to photoexcited carriers in the far-infrared region. ${ }^{10-15}$ In conventional optical-pump and THz-probe (OPTP) spectroscopy, a set of two translational delay stages is required to vary the time delay between the $\mathrm{THz}$ and probe pulses, as well as between the pump and $\mathrm{THz}$ pulses. Using this method, the time evolution of the transmittance change of materials, i.e., $\Delta T(t) / T_{0}$, can be measured. However, in this method, the effect of long-lived carriers, whose lifetime is longer than the period between successive pump pulses (typically $1 \mathrm{~ms}$ for the use of a regenerative amplifier), may distort the $\mathrm{THz}$ dielectric response, and therefore, an absolute value of the $\mathrm{THz}$ transmittance $(T)$ has not been accurately determined. ${ }^{10,11}$ The use of single-shot THz-TDS spectroscopy ${ }^{16-22}$ is essential for revealing accurate $\mathrm{THz}$ dielectric response due to the photoexcited carriers, while avoiding the artificial response due to the long-lived carriers.

In the present study, we employed a single-shot OPTP spectroscopy with an echelon mirror (step width: $20 \mu \mathrm{m}$, step height: $2.5 \mu \mathrm{m}$, and total steps: 750 steps). ${ }^{21}$ Since this technique produced an absolute value of the $\mathrm{THz}$ transmittance with a good signal-to-noise ratio, we could evaluate

${ }^{a)}$ Email: katayama@ynu.ac.jp important physical quantities, such as the complex dielectric constant, plasma frequency, and damping rate, in the $\mathrm{THz}$ region in a Si plate based on a Drude model analysis.

(a) for

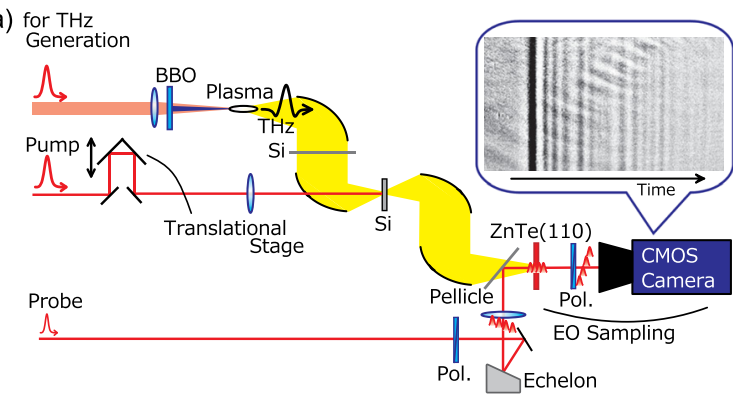

(b) for $\mathrm{THz}$

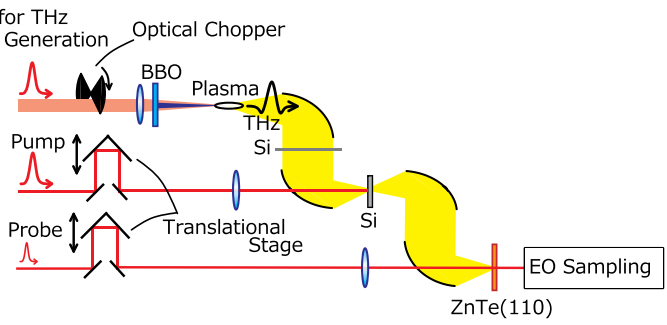

(c)

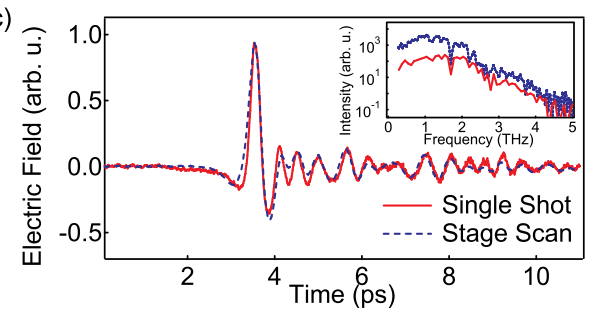

FIG. 1. Experimental setups of two OPTP spectroscopic techniques: (a) single-shot scheme and (b) conventional stage-scan scheme. The inset of (a) shows a typical image of the temporal profile of the $\mathrm{THz}$ waveform captured by a CMOS camera. (c) Time-based plots of the generated $\mathrm{THz}$ waveforms measured via the single-shot (solid curve) and stage-scan (dashed curve) schemes. The inset shows the FT power spectra obtained with both schemes. Note that the intensity under the single-shot scheme is shifted by 10 times to clearly identify the two spectra. 
Figure 1 illustrates the experimental setups for two OPTP spectroscopic techniques: (a) single-shot and (b) conventional stage-scan schemes. In both schemes, a Tisapphire regenerative amplifier system (center wavelength: $800 \mathrm{~nm}$, pulse duration: $100 \mathrm{fs}$, energy: $1.6 \mathrm{~mJ} / \mathrm{pulse}$, and repetition frequency: $1 \mathrm{kHz}$ ) was used as a light source. A commercial $750-\mu \mathrm{m}$-thick Si plate $(001)(>1500 \Omega \mathrm{cm})$ was used as a specimen. A $\mathrm{THz}$ pulse was generated from an asymmetric plasma ionization process induced by a twocolor $\left(800\right.$ and $400 \mathrm{~nm}$ ) laser field. ${ }^{23-27}$ The generated $\mathrm{THz}$ pulse was irradiated on a Si plate and was then directed to a 2-mm-thick ZnTe (110) crystal via off-axis parabolic mirrors. Subsequently, the $\mathrm{THz}$ waveform transmitted through the Si plate was measured by electro-optic (EO) sampling. The Gaussian-shaped pump and THz pulses were illuminated perpendicular to the Si plate as shown in Figs. 1(a) and 1(b). The spot size of the THz pulse was $500 \mu \mathrm{m}$, while that of the pump pulse was $3 \mathrm{~mm}$ to cover the whole area of the $\mathrm{THz}$ pulse. The absolute value of the $\mathrm{THz}$ transmittance of the $\mathrm{Si}$ plate under photoexcitation by the $800 \mathrm{~nm}$ pump was normalized compared with that without a Si plate.

In the case of the conventional OPTP scheme shown in Fig. 1(b), the transmitted $\mathrm{THz}$ waveform at a given delay time between the pump and $\mathrm{THz}$ pulses was measured with multiple delayed optical probe pulses relative to the $\mathrm{THz}$ pulse whose timing was tuned by scanning a translational stage. During the measurement, the irradiation of the pump pulses was repeated many times with $1 \mathrm{kHz}$ repetition rate. In the case of the single-shot OPTP scheme shown in Fig. 1(a), on the other hand, the $\mathrm{THz}$ waveform was detected with a single optical probe pulse with spatially encoded time-delays produced by an echelon mirror. ${ }^{21}$ Therefore, the time interval between the pump pulses could be much longer than the repetition period of the incident laser, which was typically set as $>5 \mathrm{~s}$ in our experiment. The image of the optical probe was then precisely focused on a two-dimensional CMOS camera, and the time evolution of the $\mathrm{THz}$ transmittance was readily visualized as shown in the inset of Fig. 1(a). By accumulating the observed signal along the vertical direction of the CMOS camera, we could obtain the $\mathrm{THz}$ waveform with a good signal-to-noise ratio.

Figure 1(c) shows a typical $\mathrm{THz}$ waveform without a specimen observed via either the single-shot or the conventional stage-scan scheme. The inset of Fig. 1(c) displays the Fourier-transformed (FT) power spectra. Using our singleshot scheme, a wide range of the transmittance in the $0.5-2.5$ $\mathrm{THz}$ range could be clearly detected, including water vapor absorption at $1.7 \mathrm{THz}$. Judging from the agreement between the THz waveforms and power spectra measured with both schemes, the single-shot scheme has enough performance to quantify the $\mathrm{THz}$ dielectric response due to photoexcited carriers in a $\mathrm{Si}$ plate. When measuring the $\mathrm{THz}$ dielectric response of the Si plate, all the measurements were performed at room temperature in a nitrogen gas atmosphere to eliminate water vapor.

Figure 2 shows the transmittance of the Si plate as a function of time with different pump fluences measured via the single-shot (solid curves) and the conventional stagescan (dashed curve) schemes. The transmittance intensity was averaged over the frequency range of $0.5-2.5 \mathrm{THz}$, since

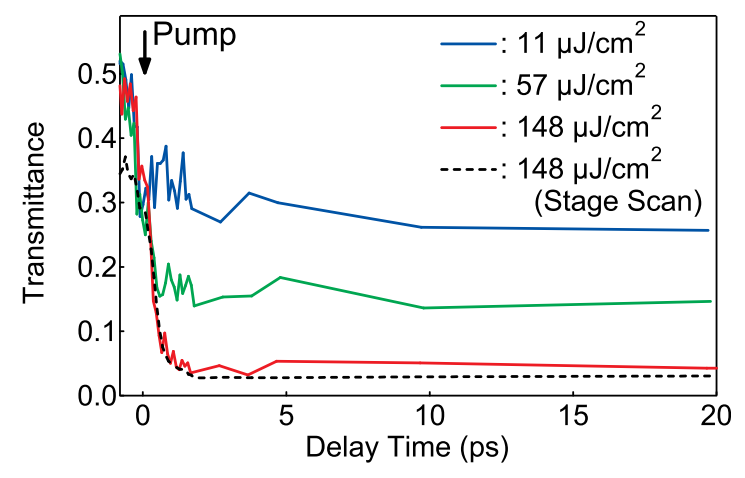

FIG. 2. Time-based plots of the THz transmittance of the Si plate at different pump fluences obtained via the single-shot (solid curves) and stage-scan (dashed curve) schemes. The optical pumping occurred at time $t=0$ as shown by the arrow.

the transmittance spectra were flat in this frequency range. ${ }^{28}$ Each transmittance curve immediately decreases at time $t=0$ just after the pump pulse illumination, and subsequently has a plateau without a decay profile, suggesting the existence of long-lived carriers. Upon increasing the fluence, the transmittance becomes lower due to the increase of photoexcited carriers. Note that, for the $148 \mu \mathrm{J} / \mathrm{cm}^{2}$ pump illumination, the transmittance values before the pump disagree between the two schemes: the values are 0.48 and 0.35 for the single-shot and the stage-scan schemes, respectively. Using the well-known refractive index of $\mathrm{Si}(=3.42)$ in the $\mathrm{THz}$ frequency region, the $\mathrm{THz}$ transmittance of the Si plate without photoexcitation was estimated to be 0.49 . This value is in a quite good agreement with that measured via the single-shot scheme. On the other hand, the transmittance observed via the stage-scan scheme gives a smaller value than the calculated one, implying an overestimation of the carrier density as a result of artifacts in the response caused by the accumulation of long-lived carriers. Indeed, in the case of the stage-scan scheme, the transmittance value before the arrival of the pump pulse became lower with increasing the pump fluence. This also suggests the existence of the artifacts due to the accumulation of long-lived carriers.

To evaluate the accurate $\mathrm{THz}$ dielectric response due to the photoexcited carriers in the Si plate, a Drude model analysis was carried out in the case of the single-shot scheme. The observed complex transmittance $\tilde{T}(\omega)$ at each angular frequency $\omega$ under the thin film approximation is expressed $\operatorname{as}^{29,30}$

$$
\tilde{T}(\omega)=\frac{n_{\mathrm{Si}}+1}{n_{\mathrm{Si}}+1-i \omega \tilde{\varepsilon} d / c},
$$

where $\tilde{\varepsilon}(\omega)$ is the complex dielectric constant

$$
\tilde{\varepsilon}(\omega)=\varepsilon_{\infty}-\frac{\omega_{\mathrm{p}}^{2}}{\omega(\omega+i \gamma)} .
$$

Here, $\varepsilon_{\infty}(=11.7$ (Ref. 31)) is the dielectric constant at the high frequency limit, $\omega_{\mathrm{p}}$ is the plasma frequency, $\gamma$ is the damping rate, $c$ is the speed of light, $n_{\mathrm{Si}}$ is the refractive index of the Si without photoexcitation, and $d(=13.5 \mu \mathrm{m})$ is the thickness of the photoexcited region estimated from the penetration depth of the pump wavelength. ${ }^{31} \mathrm{We}$ assumed 
that the photoexcitation uniformly occurred in the region. The plasma frequency is given by

$$
\omega_{\mathrm{p}}=\sqrt{\frac{n e^{2}}{\varepsilon_{0} m^{*}}},
$$

where $n$ is the carrier density, $m^{*}\left(=0.26 m_{0}\right.$ (Ref. 31)) is the effective mass, $e$ is the elementary charge, and $\varepsilon_{0}$ is the dielectric constant of vacuum. From these equations, we expect that the transmittance will become lower as a result of either increasing the plasma frequency or decreasing the damping rate.

Figure 3(a) shows the real (solid circles) and imaginary (open circles) parts of the dielectric constant at time $t=+4 \mathrm{ps}$

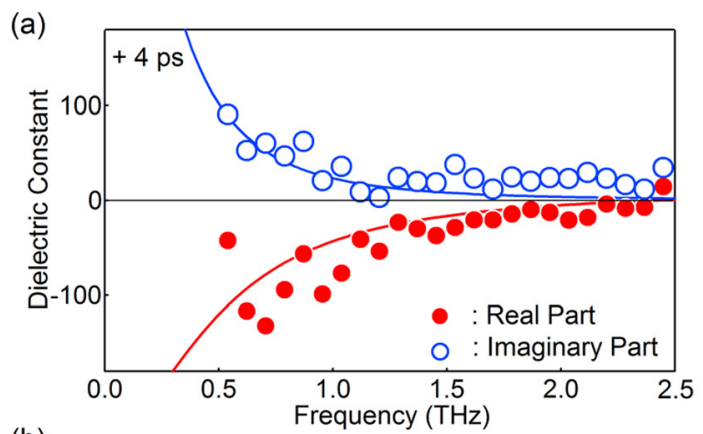

(b)

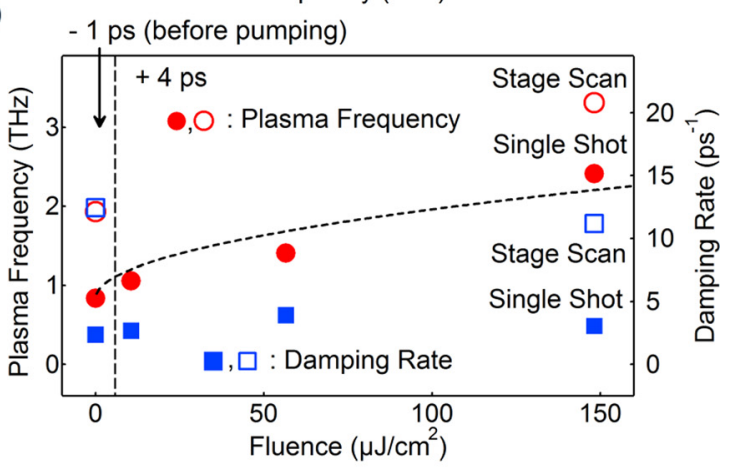

(c)

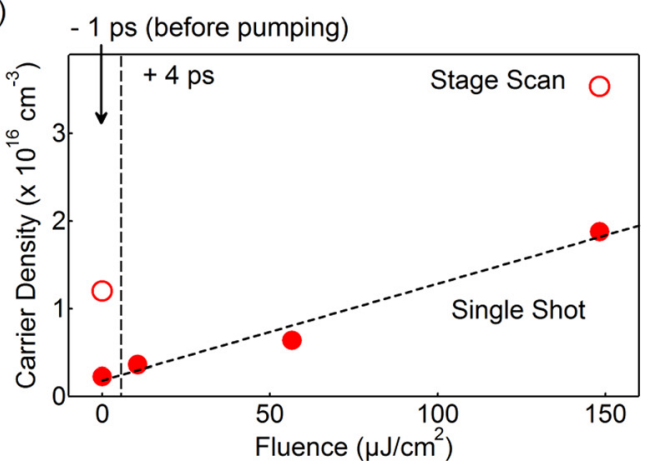

FIG. 3. (a) Real (solid circles) and imaginary (open circles) parts of the dielectric constant at time $t=+4$ ps with the pump fluence of $148 \mu \mathrm{J} / \mathrm{cm}^{2}$ measured via the single-shot scheme. The solid curves are the best fits, derived from Eq. (2) in the main text. (b) The estimated plasma frequency (solid circles) and damping rate (solid squares) as a function of the pump fluences under the single-shot scheme. The values of the plasma frequency and damping rate before pumping are estimated from the $\mathrm{THz}$ transmittance at time $t=-1 \mathrm{ps}$. The dashed curve shows a square root relationship between the plasma frequency and the pump fluence. (c) The estimated carrier density as a function of the pump fluence obtained with the single-shot scheme (solid circles). The carrier density is proportional to the pump fluence as shown by a dashed line. For the case without pumping, the plasma frequency, damping rate, and the carrier density estimated under the stage-scan scheme are also shown by open circles and a square in (b) and (c). with the pump fluence of $148 \mu \mathrm{J} / \mathrm{cm}^{2}$, measured via the single-shot scheme. The solid curves are the best fits derived from Eq. (2) with two adjustable parameters: $\omega_{\mathrm{p}}=2.4 \mathrm{THz}$ and $\gamma=3.0 \mathrm{ps}^{-1}$. Because both the real and imaginary parts of the dielectric constant were dispersive under the pump illumination, we could accurately estimate the two physical quantities. Figure 3(b) shows the estimated plasma frequency (solid circles) and damping rate (solid squares) as a function of pump fluence. To evaluate these physical quantities without pumping, we also display the values of the plasma frequency and damping rate before pumping, which are estimated at time $t=-1$ ps. As we would expect from Eq. (3), the plasma frequency is proportional to the square root of the pump fluence, i.e., the carrier density. On the other hand, the damping rate is almost independent of the pump fluence, suggesting that neither carrier-carrier collisions nor Auger recombination contributes to the damping rate, and that carrier-phonon scattering might play an important role in the damping. ${ }^{8}$ Figure 3(c) shows the estimated carrier density as a function of the pump fluence. As shown in the figure, the carrier density linearly increases with the fluence as expected. The carrier density without pumping was estimated as $n<2.3 \times 10^{15} \mathrm{~cm}^{-3}$. 32 Under the optical pumping with the fluence of $148 \mu \mathrm{J} / \mathrm{cm}^{2}$, the carrier density was $n=1.9 \times 10^{16} \mathrm{~cm}^{-3}$, and as a result, the net increase of the carrier density via the photoexcitation was estimated to be $\Delta n \sim 1.7 \times 10^{16} \mathrm{~cm}^{-3}$.

Finally, we compare the physical quantities (plasma frequency, damping rate, and carrier density) estimated under the single-shot scheme with those under the conventional stagescan scheme in the case without pumping. The physical quantities estimated under the stage-scan scheme, $\omega_{\mathrm{p}}=1.9 \mathrm{THz}$ and $\gamma=12 \mathrm{ps}^{-1}$, as well as $n=1.2 \times 10^{16} \mathrm{~cm}^{-3}$, are denoted by an open square and open circles in Figs. 3(b) and 3(c). These values are anomalously high for the Si plate, probably due to photoexcited carriers that survived for more than $1 \mathrm{~ms}$. Since the pump pulses arrive at the specimen once per millisecond in the stage-scan method, the long-lived carriers optically generated by previous pump pulses are accumulated, ${ }^{8}$ leading to the overestimation of the physical quantities. Here, we estimated the lifetime $(\tau)$ of the long-lived carriers, assuming that the lifetime shows an exponential decay. In the case of the conventional stage-scan scheme, the optical pumping was repeated every $1 \mathrm{~ms}(\Delta t=1 \mathrm{~ms})$. Therefore, the carrier density observed via the stage-scan scheme under the $148 \mu \mathrm{J} / \mathrm{cm}^{2}$ pump illumination, $n\left(=3.5 \times 10^{16} \mathrm{~cm}^{-3}\right)$, is expressed as

$$
n=n_{0}+\sum_{m=0}^{\infty} \Delta n \exp (-m \Delta t / \tau)=n_{0}+\Delta n \frac{1}{1-\exp (-\Delta t / \tau)},
$$

where $n_{0}$ and $\Delta n$ are the carrier density before pumping $\left(n_{0}<2.3 \times 10^{15} \mathrm{~cm}^{-3}\right)$ and the net increase of the carrier density $\left(\Delta n \sim 1.7 \times 10^{16} \mathrm{~cm}^{-3}\right)$, respectively, observed via the single-shot scheme, as shown in Fig. 3(c). Using this simple calculation, $\tau$ was estimated to be $1.4 \mathrm{~ms}$ whose value is quite in a good agreement with the value obtained in Ref. 8.

In contrast, our single-shot scheme enables accurate observation of the dielectric response, while avoiding any artificial response due to the accumulation of long-lived 
photoexcited carriers. These results clearly indicate that the use of single-shot OPTP spectroscopy is indispensable for providing quantitative measurement of the $\mathrm{THz}$ dielectric response of materials when long-lived carriers exist.

In conclusion, we have demonstrated the $\mathrm{THz}$ dielectric response due to photoexcited carriers in a Si plate via singleshot OPTP spectroscopy with an echelon mirror. In contrast to conventional stage-scan OPTP spectroscopy, this technique allows single-shot detection of the $\mathrm{THz}$ response of materials at a given delay time between the pump and the $\mathrm{THz}$ pulses. Since the time interval was sufficiently spaced between the pump pulses, we could achieve accurate observation of the $\mathrm{THz}$ transmittance of the Si plate with different pump fluences, while avoiding the pileup effect due to the photoexcited long-lived carriers. Based on a Drude model analysis, the optically induced complex dielectric constant, plasma frequency, and damping rate in the $\mathrm{THz}$ region were quantitatively evaluated.

This work was supported in part by the Grants-in-Aid for Scientific Research (KAKENHI, Nos. 23241034, 26107517, and 15K13378) from the Japan Society for the Promotion of Science (JSPS). I.K. acknowledges financial support from Strategic Information and Communications R\&D Promotion Programme (SCOPE, No. 145003103) of Japan Ministry of Internal Affairs and Communications.

${ }^{1}$ C. H. Kao, H. Chen, S. Z. Chen, Y. J. Chen, and Y. C. Chu, Thin Solid Films 570, 564 (2014).

${ }^{2}$ J. Fan, C. Huang, and L. Zhu, Opt. Express 23, 2973 (2015).

${ }^{3}$ M. Elzouka and S. Ndao, Appl. Phys. Lett. 105, 243510 (2014).

${ }^{4}$ J. A. Moon and J. Tauc, J. Appl. Phys. 73, 4571 (1993).

${ }^{5}$ A. J. Sabbah and D. M. Riffe, Phys. Rev. B 66, 165217 (2002).

${ }^{6}$ K. E. Myers, Q. Wang, and S. L. Dexheimer, Phys. Rev. B 64, 161309(R) (2001).

${ }^{7}$ V. Klimov, D. McBranch, and V. Karavanskii, Phys. Rev. B 52, R16989(R) (1995).

${ }^{8}$ Y. Arafat, F. M. Mohammedy, and M. M. S. Hassan, Int. J. Optoelectron. Eng. 2, 5 (2012).
${ }^{9}$ W. Shockley and W. T. Read, Phys. Rev. 87, 835 (1952).

${ }^{10} \mathrm{G}$. Li, D. Li, Z. Jin, and G. Ma, Opt. Commun. 285, 4102 (2012).

${ }^{11}$ P. U. Jepsen, W. Schairer, I. H. Libon, U. Lemmer, N. E. Hecker, M. Birkholz, K. Lips, and M. Schall, Appl. Phys. Lett. 79, 1291 (2001).

${ }^{12}$ K. P. H. Lui and F. A. Hegmann, Appl. Phys. Lett. 78, 3478 (2001).

${ }^{13}$ H. F. Tiedje, H. K. Haugen, and J. S. Preston, Opt. Commun. 274, 187 (2007).

${ }^{14}$ M. Tsubouchi, M. Nagai, and Y. Ohshima, Opt. Lett. 37, 3528 (2012).

${ }^{15}$ A. Beaudoin, B. Salem, T. Baron, P. Gentile, and D. Morris, Phys. Rev. B 89, 115316 (2014).

${ }^{16}$ Z. Jiang and X.-C. Zhang, Appl. Phys. Lett. 72, 1945 (1998).

${ }^{17}$ J. Shan, A. S. Weling, E. Knoesel, L. Bartles, M. Bonn, A. Nahata, G. A. Reider, and T. F. Heinz, Opt. Lett. 25, 426 (2000).

${ }^{18}$ B. Yellampalle, K. Y. Kim, G. Rodriguez, J. H. Glownia, and A. J. Taylor, Appl. Phys. Lett. 87, 211109 (2005).

${ }^{19}$ H. Murakami, K. Shimizu, M. Katsurada, and S. Nashima, J. Appl. Phys. 104, 103111 (2008).

${ }^{20}$ K. Y. Kim, B. Yellampalle, J. H. Glownia, A. J. Taylor, and G. Rodriguez, Phys. Rev. Lett. 100, 135002 (2008).

${ }^{21}$ Y. Minami, Y. Hayashi, J. Takeda, and I. Katayama, Appl. Phys. Lett. 103, 051103 (2013).

${ }^{22}$ S. M. Teo, B. K. Ofori-Okai, C. A. Werley, and K. A. Nelson, Rev. Sci. Instrum. 86, 051301 (2015).

${ }^{23}$ M. D. Thomson, V. Blank, and H. G. Roskos, Opt. Express 18, 23173 (2010).

${ }^{24}$ Y. Minami, M. Nakajima, and T. Suemoto, Phys. Rev. A 83, 023828 (2011).

${ }^{25}$ E. Matsubara, M. Nagai, and M. Ashida, Appl. Phys. Lett. 101, 011105 (2012).

${ }^{26}$ Y. Minami, T. Kurihara, K. Yamaguchi, M. Nakajima, and T. Suemoto, Appl. Phys. Lett. 102, 151106 (2013).

${ }^{27}$ Y. Minami, T. Kurihara, K. Yamaguchi, M. Nakajima, and T. Suemoto, Appl. Phys. Lett. 102, 041105 (2013).

${ }^{28}$ F. D. J. Brunner, A. Schneider, and P. Günter, Opt. Express 17, 20684 (2009).

${ }^{29}$ J. J. Tu, C. C. Homes, and M. Strongin, Phys. Rev. Lett. 90, 017402 (2003).

${ }^{30}$ K. Yokota, J. Takeda, C. Dang, G. Han, D. N. McCarthy, T. Nagao, S. Hishita, M. Kitajima, and I. Katayama, Appl. Phys. Lett. 100, 251605 (2012).

${ }^{31}$ E. D. Palik, Handbook of Optical Constants of Solids (Academic Press, New York, 1985), Chap. 11.

${ }^{32}$ Without pump illumination, the real and imaginary parts of the dielectric constant had non-dispersive shapes in their spectra due to small number of carriers. Therefore, we could only estimate the maximum range of the carrier density, i.e., $n_{0}<2.3 \times 10^{15} \mathrm{~cm}^{-3}$. 